[4] J. H. McClellan, "The design of two-dimensional digital filters by transformations," in Proc. 7th Annu. Princeton Conf. Inform. Sci. Syst., 1973, pp. 247-251.

[5] R. M. Mersereau, W. G. Mecklenbráuker, and T. F. Quatieri, "McClellan transformations for two-dimensional digital filtering: I-design," IEEE Trans. Circuits Syst., vol. CAS-23, pp. 405-414, July 1976.

[6] W. G. Mecklenbráuker and R. M. Mersereau, "McClellan transformations for two-dimensional digital filtering: Ii-implementation," IEEE Trans. Circuits Syst., vol. CAS-23, pp. 414-422, July 1976.

[7] D. Hale, "3-D depth migration via McClellan transformations," Geophys., vol. 56, pp. 1778-1785, Nov. 1991.

[8] J. H. McClellan, "On the design of one-dimensional and twodimensional FIR digital filters," Ph.D. dissertation, Rice Univ., Hiouston, TX, Apr. 1973.

[9] R. M. Mersereau, "The design of arbitrary 2-D zero phase FIR filters using transformations," IEEE Trans. Circuits Syst., vol. CAS-27, pp. 142-144, Feb. 1980

[10] J. H. McClellan and D. S. Chan, "A 2-D filter structure derived from the Chebyshev recursion," IEEE Trans. Circuits Syst., vol. CAS-24, pp. 372-378, 1977.

[11] L. J. Karam, "Design of complex digital FIR filters in the Chebyshev sense," Ph.D. dissertation, Georgia Inst. Technol., Atlanta, Mar. 1995.

\section{A Study of Cramer-Rao Bounds on Object Shape Parameters From Scattered Field}

\author{
Prabhakar S. Naidu and A. Buvaneswari
}

\begin{abstract}
We have studied the Cramér-Rao bounds (CRB's) on size and orientation of a known object using the scattered (acoustic or electromagnetic) field. A circular array of transievers surrounding the object under test is proposed. The CRB's are theoretically derived and compared with the mean square errors computed via Monte Carlo simulations. The CRB's are found to increase rapidly with wavelength. For reliable estimates, the wavelength of illumination must be smaller than the size of the object.
\end{abstract}

\section{INTRODUCTION}

In diffraction tomography, the scattered wavefield (acoustic, optical, or electromagnetic) is used for reconstruction or imaging of an object, that is, to estimate the spatial variation of the refractive index or some other parameter responsible for scattering [1]. When the object is known, the information of interest is its actual location, size, and orientation. Using the diffraction tomography, the location parameters of a known object have been estimated [2]-[6]. Devaney and Tsihrintiz [2] have considered the maximum likelihood (ML) estimation of location parameters from a weakly scattering object. The extension of this work to strongly scattering objects has been done by the same authors in [3], and the experimental verification has been reported in [4]. The Cramér-Rao bounds (CRB's) on the location estimates, when the evanescent waves are considered, for strongly scattering objects have been derived by Schatzberg et al. [12]. In addition to location parameters, the problem of

Manuscript received November 27, 1995; revised November 2, 1998. The associate editor coordinating the review of this paper and approving it for publication was Dr. Yun Q. Shi.

The authors are with the Department of Electrical and Computer Engineering, Indian Institute of Science, Bangalore, India.

Publisher Item Identifier S 1053-587X(99)03260-2. estimating the scale and rotation parameters of an object are of great significance in some applications, such as in geophysical exploration, underwater imaging, and medical and industrial imaging, where diffraction tomography is more appropriate. Using projection data in the context of x-ray tomography, Rossi and Wilsky [5] have proposed the estimation of the location of a simple object. Later, Rossi and Wilsky [6] and Spielman [13] proposed ML estimation of the size, orientation, and elongation of an elliptic disk. They conclude that the size and orientation are determined quite reliably, especially for very eccentric objects. In the present correspondence, we derive the CRB's on scale and orientation of a weakly scattering object (for which the Born approximation holds) from the noisy data obtained by using a circular array of transceivers. Simulation results show that for a class of polygonal objects, the shape parameters can be estimated more reliably for an eccentric object, viz., a rectangular strip, and when the object is large compared with the wavelength.

\section{Diffraction TOMOgRaphic IMAging Using a CIRCUlar ARRAY}

Diffraction tomography refers to the cross sectional imaging of objects from diffracted or scattered wavefields, which may be either electromagnetic or acoustic. The array of receivers employed in tomographic imaging is usually a linear array, but sometimes, a circular array is also used. In [7], the authors used a circular array to image a weakly scattering object show that it performs better than a linear array. In polar coordinates and under far-field approximation, the scattered wavefield is related to the Fourier transform of the object [7]

$$
\begin{aligned}
& O\left(k_{0}\left(\cos \chi-\cos \chi_{0}\right), k_{0}\left(\sin \chi-\sin \chi_{0}\right)\right) \\
& \quad=\frac{4 \sqrt{p_{0}}}{k_{0}^{2}} e^{-j\left(k_{0} R-(\pi / 4)\right)} \sqrt{\frac{\pi k_{0} R}{2}} \psi_{s}\left(R, \chi, \chi_{0}\right) .
\end{aligned}
$$

In this equation, $O\left(k_{0}\left(\cos \chi-\cos \chi_{0}\right), k_{0}\left(\sin \chi-\sin \chi_{0}\right)\right.$ refers to the two-dimensional (2-D) Fourier transform of the object evaluated at $k_{s}=k_{0}\left(\cos \chi-\cos \chi_{0}\right)$ and $k_{y}=k_{0}\left(\sin \chi-\sin \chi_{0}\right)$, where $\left(k_{x}, k_{y}\right)$ are the spatial frequency coordinates, $\psi_{s}\left(R, \chi, \chi_{0}\right)$ is the scattered field received at a sensor at angle of $\chi$ with the reference direction, and the angle of illumination is $\chi_{0}$ (see Fig. 1 for illustration). $\sqrt{p_{0}}$ is the amplitude of illumination, and $k_{0}$ is the wavenumber of illumination. As $\chi$ is varied from 0 to $2 \pi$, the point $\left(k_{x}, k_{y}\right)$ executes a circle of radius $2 k_{0}$ and centered at $\left(-k_{0} \cos \chi_{0},-k_{0} \sin \chi_{0}\right)$. Next, as we vary $\chi_{0}$ from 0 to $2 \pi$, the circle sweeps a disc of radius $2 k_{0}$. Hence, the 2-D Fourier transform of the object lying within a disc of size $2 k_{0}$ is available for shape estimation.

\section{MAXimum LiKelihood Estimation}

Let $o_{0}(x, y)$ represent a standard object and $o(x, y)$ represent scaled by a factor $s$ and rotated through an angle $\beta$. They are related as

$$
o(x, y)=o_{0}\left(\frac{x \cos (\beta)+y \sin (\beta)}{s}, \frac{y \cos (\beta)-x \sin (\beta)}{s}\right)
$$

Let the observed field be a sum of scattered field and spatially uncorrelated Gaussian noise.

$$
y\left(\chi, \chi_{0}\right)-\psi_{S}\left(\chi, \chi_{0} ; s, \beta\right)+n\left(\chi, \chi_{0}\right)
$$




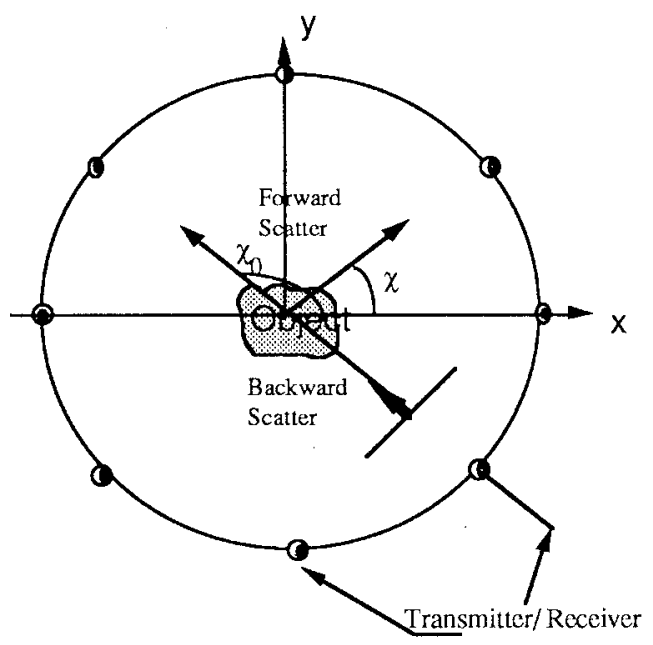

(a)

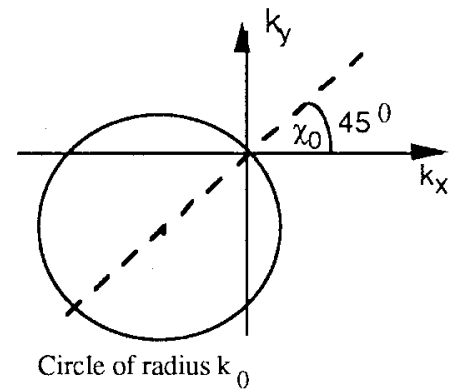

(b)

Fig. 1. (a) Array of transreceivers is used to illuminate an object and to receive the scattered field. $\chi$ : angular coordinate of a sensor. $\chi_{0}$ : angle of incidence. (b) For $\chi_{0}=45^{\circ}$, the scattered field received by a circular array is proportional to the object Fourier transform on a circle as shown.

Since the noise is assumed to be spatially uncorrelated Gaussian, the ML estimator of the object parameters $s$ and $\beta$ can be obtained through maximization of the likelihood function given by

$$
L(s, \beta)=\frac{-1}{2 \sigma_{n}^{2}} \sum_{\chi_{0}} \int_{0}^{2 \pi}\left|y\left(\chi, \chi_{0}\right)-\psi_{s c}\left(\chi, \chi_{0}, s, \beta\right)\right|^{2} R d \chi .
$$

In (3), we use the expression for the scattered field $\psi_{s c}\left(\chi, \chi_{0}, s, \beta\right)$ from (1) in terms of the object Fourier transform $\psi_{s c}\left(\chi, \chi_{0} ; s, \beta\right)=\gamma s^{2} O_{0}\left(s k_{x}^{\prime}, s k_{y}^{\prime}\right)$, where $O_{0}\left(k_{x}, k_{y}\right)$ is the Fourier transform of standard object $(s=1$ and $\beta=0), \gamma=\left(k_{0}^{2} / 4\right) \sqrt{\left(2 p_{0} / \pi k_{0} R\right)} e^{j\left(k_{0} R+(\pi / 4)\right.}$, $k_{x}=k_{0}\left\{\cos (\chi-\beta)-\cos \left(\chi_{0}-\beta\right)\right\}$, and $k_{y}=$ $k_{0}\left\{\sin (\chi-\beta)-\sin \left(\chi_{0}-\beta\right)\right\}$. Note that the scaled and rotated object function may be derived from the original object function as $o(x, y)=o_{0}((x \cos \beta+y \sin \beta) / s,(-x \cos \beta+y \sin \beta) / s)$. To estimate the unknown parameters, we need to maximize the likelihood function given by (3). A coarse scan is first performed, and the maximum point is noted. With this as a starting point, the true maximum is reached using the Simplex algorithm [10].

\section{CRAMÉR-RaO Bounds}

The CRB's on the variance of the estimated parameters may be obtained from the Fisher information matrix, which is defined as

$$
\left[\boldsymbol{J}_{i, j}=-E\left\{\frac{\partial^{2} L\left(x_{i}, x_{j}\right)}{\partial x_{i} \partial x_{j}}\right\}\right.
$$

where $x_{1}=s, x_{2}=\beta$. The CRB's are given by the inverse of the Fisher information matrix $E\left\{\boldsymbol{x} \boldsymbol{x}^{H}\right\} \geq \boldsymbol{J}^{-1}$, where $\boldsymbol{x}=\operatorname{col}\{s, \beta\}$. The Fisher information matrix is easily computed by differentiating the likelihood function with respect to $s$ and $b$ and then carrying out the expectation operation. After some tedious algebra, we obtain

$$
\boldsymbol{J}^{-1}=-\left[\begin{array}{cc}
\frac{b}{a b-c^{2}} & \frac{-c}{a b-c^{2}} \\
\frac{-c}{a b-c^{2}} & \frac{a}{a b-c^{2}}
\end{array}\right]
$$

where

$$
\begin{aligned}
a= & E\left\{\frac{\partial^{2} L}{\partial s^{2}}\right\}=-\frac{|\gamma|^{2}}{\sigma_{n}^{2}} \operatorname{Re} \sum_{k_{z}^{\prime}, k_{y}^{\prime}} \mid 2 s O_{0}\left(s k_{x}^{\prime}, s k_{y}^{\prime}\right) \\
& +s^{2} k_{x}^{\prime} \frac{\partial O_{0}\left(s k_{x}^{\prime}, s k_{y}^{\prime}\right)}{\partial\left(s k_{x}^{\prime}\right)}+\left.s^{2} k_{y}^{\prime} \frac{\partial O_{0}\left(s k_{x}^{\prime}, s k_{y}^{\prime}\right)}{\partial\left(s k_{y}^{\prime}\right)}\right|^{2} \\
b= & E\left\{\frac{\partial^{2} L}{\partial \beta^{2}}\right\}=-\frac{|\gamma|^{2}}{\sigma_{n}^{2}} s^{2} \operatorname{Re} \sum_{k_{x}^{\prime}, k_{y}^{\prime}} \\
& \cdot\left|s k_{y}^{\prime} \frac{\partial O_{0}\left(s k_{x}^{\prime}, s k_{y}^{\prime}\right)}{\partial\left(s k_{x}^{\prime}\right)}-s k_{x}^{\prime} \frac{\partial O_{0}\left(s k_{x}^{\prime}, s k_{y}^{\prime}\right)}{\partial\left(s k_{y}^{\prime}\right)}\right|^{2} \\
c= & -\frac{|\gamma|^{2}}{\sigma_{n}^{2}} s^{3} \sum_{k_{x}^{\prime}} \sum_{k_{y}^{\prime}}\left(s k_{y}^{\prime} \frac{\partial O_{0}\left(s k_{x}^{\prime}, s k_{y}^{\prime}\right)}{\partial\left(s k_{x}^{\prime}\right)}-s k_{x}^{\prime} \frac{\partial O_{0}\left(s k_{x}^{\prime}, s k_{y}^{\prime}\right)}{\partial\left(s k_{y}^{\prime}\right)}\right)^{*} . \\
& \left(\left(2 O_{0}\left(s k_{x}^{\prime}, s k_{y}^{\prime}\right)+s k_{x}^{\prime}\right.\right. \\
& \left.\left.\cdot \frac{\partial O_{0}\left(s k_{x}^{\prime}, s k_{y}^{\prime}\right)}{\partial\left(s k_{x}^{\prime}\right)}+s k_{y}^{\prime} \frac{\partial O_{0}\left(s k_{x}^{\prime}, s k_{y}^{\prime}\right)}{\partial\left(s k_{y}^{\prime}\right)}\right)\right) .
\end{aligned}
$$

When the object is real and symmetric about the $y$ axis, $O_{0}\left(k_{x}^{\prime}, k_{y}^{\prime}\right)=O_{0}\left(k_{x}^{\prime},-k_{y}^{\prime}\right)$; then, each term in the expression for $c$, after it is expanded, becomes an odd function in $k_{y}^{\prime}$. As an example, the first term in the product is $2 s k_{y}^{\prime} O_{0}\left(s k_{x}^{\prime}, s k_{y}^{\prime}\right)\left(\partial O_{0}^{*}\left(s k_{x}^{\prime}, s k_{y}^{\prime}\right) / \partial s k_{x}^{\prime}\right)$, which has an odd symmetry in $k_{y}^{\prime}$ that, when summed over all $k_{y}^{\prime}$, will vanish. Thus, the cross term goes to zero for an object having symmetry about one axis. It was observed by numerical evaluation that $c \ll a$ and $b$ even for a finite number of receivers and illuminations, for example, for 64 sensors and four illuminations, $c$ is approximately $2 \%$ of $a$ and $b$. Finally, the CRB's on scale and orientation are approximately given by (6) and (7), shown at the bottom of the next page, where $\left(|\gamma|^{2} / \sigma_{n}^{2}\right)=\left(p_{0} / \sigma_{n}^{2}\right)\left(k_{0}^{3} / 8 \pi R\right)=\operatorname{SNR}\left(k_{0}^{3} / 8 \pi R\right)$ is proportional to the source SNR, which is defined as a ratio of the power radiated by the transmitter to the average noise power in all sensors. Since we are dealing with weak scattering, the scattered power is much smaller, to the order $10^{-4}$. Therefore, it is more appropriate to define $\mathrm{SNR}_{s c}$ as a ratio of average scattered power intercepted by array to average noise power. Note that $\mathrm{SNR}_{s c}$ is about $38 \mathrm{~dB}$ below the source SNR.

The dependence of CRB's on wavelength is shown in Fig. 2. We have assumed four illuminations and a large number of sensors 


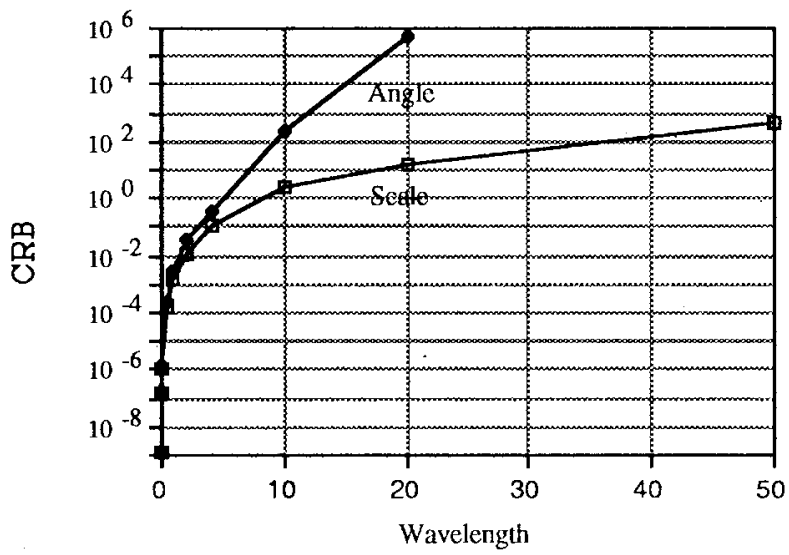

Fig. 2. CRB's as a function of wavelength (in meters) for a square object $\left(2 \times 2 \mathrm{~m}^{2}\right)$.

(8192) so that the result is practically independent of the number of sensors. The standard object is a square of size $2 \times 2 \mathrm{~m}^{2}$. The scale factor $s=2$, and orientation $\beta=(\pi / 9)$. From Fig. 2, we observe that the CR bounds for both parameters increase with increasing wavelength. For wavelengths much greater than the object size, we may conclude that since the CRB's are too high particularly for orientation, no meaningful estimate of the scale or orientation can be made. Intuitively, it would appear that the object behaves as an isotropic point scatterer when the object size is a fraction of the wavelength.

\section{Simulation StUdies}

For computer simulation studies, we have considered a weakly scattering (refractive index contrast $=0.01)$ square cylinder $(4 \times$ $4 \mathrm{~m}^{2}$ ) placed at the center of a circular (radius $=100 \mathrm{~m}$ ) array of transceivers. It is rotated $\pi / 9 \mathrm{rad}\left(20^{\circ}\right)$ with respect to the north. Each transmitter radiates a monochromatic radiation, and all receivers, except the one at the transmitter, receive the scattered field that was computed using Fourier transform approach. To each sensor output, adequate white noise is added so that the ratio of the average scattered power to the noise power is equal to the desired $\mathrm{SNR}_{s c}$. A likelihood function was created for a $2 \times 2 \mathrm{~m}^{2}$ square object with zero orientation as a standard object. The likelihood function was then computed as a function of the scale factor and orientation over a grid of discrete values. From the position of maximum on the grid, using the Simplex algorithm, the true maximum was reached. Thus, the ML estimates of the scale and orientation were obtained.

To verify the CRB's evaluated in the last section, we have carried out a numerical experiment where ML estimates of the scale and orientation were carried out on 50 independent data sets that were obtained by adding independent white Gaussian noise to the scattered field (see Table I). Further, at $12 \mathrm{~dB} \mathrm{SNR}_{s c}$, the bias in the estimate of scale and orientation was found to be negligible. This justifies our assumption of unbiased estimate required for the validity of $\mathrm{CR}$ bounds [11].
TABLE I

Comparison of Mean Square Error (MSE) and CRB's. The WaVelength OF THE RADIATION IS ASSUMED TO BE $1 \mathrm{~m}$. NL: NUMBER OF ILLUMinATIONS. NR: Number of Sensors. $s=2, \beta=20^{\circ}$, SNR $=12.5 \mathrm{~dB}$, SQuare ObJeCt. Fifty Monte-Carlo Runs Were Performed (Also in Tables II AND III)

\begin{tabular}{r|l|l|l|l|l}
\hline NL & NR & CRB for Scale & $\begin{array}{c}\text { MSE in } \\
\text { estimated scale }\end{array}$ & $\begin{array}{c}\text { CRB for } \\
\text { orientation }\end{array}$ & $\begin{array}{c}\text { MSE in } \\
\text { estimated } \\
\text { onentation }\end{array}$ \\
\hline 8 & 32 & $7.6981 \mathrm{e}-05$ & $8.7727 \mathrm{e}-05$ & $1.5914 \mathrm{e}-04$ & $1.6827 \mathrm{e}-04$ \\
\hline 8 & 64 & $4.2277 \mathrm{e}-05$ & $4.4877 \mathrm{e}-05$ & $8.7171 \mathrm{e}-05$ & $8.9678 \mathrm{e}-05$ \\
\hline 16 & 32 & $3.9991 \mathrm{e}-05$ & $4.3675 \mathrm{e}-05$ & $7.9927 \mathrm{e}-05$ & $8.1571 \mathrm{e}-05$ \\
\hline 16 & 64 & $2.1284 \mathrm{e}-05$ & $2.1890 \mathrm{e}-05$ & $4.3235 \mathrm{e}-05$ & $4.4158 \mathrm{e}-05$ \\
\hline 32 & 32 & $2.0207 \mathrm{e}-05$ & $2.1674 \mathrm{e}-05$ & $4.3350 \mathrm{e}-05$ & $4.5057 \mathrm{e}-05$ \\
\hline 32 & 64 & $1.0605 \mathrm{e}-05$ & $1.1256 \mathrm{e}-05$ & $2.2128 \mathrm{e}-05$ & $2.3346 \mathrm{e}-05$ \\
\hline
\end{tabular}

The estimation of orientation of an object is generally more difficult than the estimation of scale; this is particularly so when the object is equidimensional, such as a square. The number of illuminations required to obtain the correct estimate of the object parameters was also studied for objects of different shape. We considered three different types of objects that were all of equal cross sectional area

a) square $(2 \mathrm{~m} \times 2 \mathrm{~m})$;

b) triangle $(4 \mathrm{~m} \times 2 \mathrm{~m})$;

c) rectangular strip $(4 \mathrm{~m} \times 1 \mathrm{~m})$.

The scattered fields for these objects were generated assuming the scale $=2$ and the orientation $=\pi / 9\left(20^{\circ}\right)$. The threshold SNR required to achieve $90 \%$ (45 successes out of 50 trials) is ploted as a function of the number of illuminations in Fig. 3. For a success, the estimated scale factor must lie within $2.0 \pm 0.2$, and orientation must lie within $(\pi / 9) \pm(\pi / 90)\left(20^{\circ} \pm 2^{\circ}\right)$. Of three objects, the strip required the lowest threshold SNR, and the square required the highest threshold SNR. This is because the strip is the least circular object. This observation is in conformity with the findings in [13], where elliptical objects in the context of x-ray tomography were studied.

To see the effect of the scale and orientation of the object on the estimation error, we have computed CRB and MSE for the following cases:

1) $s=2.0$ with $\beta=0,10,20$, and $40^{\circ}$; (other parameters: 16 illuminations, 32 receivers, $\mathrm{SNR}_{s c}=15.3 \mathrm{~dB}$ );

2) $\beta=20^{\circ}$ with $s=0.25,0.5,1.0,2.0$, and 3.0; (other parameters: 16 illuminations, 32 receivers, $\mathrm{SNR}_{s c}=15.3 \mathrm{~dB}$ ).

We note that the orientation angle of the object does not have a significant effect on the estimation of scale (Table II); however, the size of the object influences the accuracy of the estimated scale. As we are comparing the MSE and CRB at different scales, it would be meaningful only if we compare the normalized or relative MSE and CRB (that is divided by $s^{2}$ ). Table III shows the MSE and CRB of orientation and $\mathrm{MSE} / \mathrm{s}^{2}$ and $\mathrm{CRB} / s^{2}$ of the scale parameter for different values of $s$. The MSE and CRB on scale and orientation decrease as $s$ increases. Intuitively, it is easy to see that for large objects, it is easy to determine these parameters. Similar findings have been reported in [13] in the context of x-ray tomography.

$$
\begin{aligned}
E\left\{(\hat{s}-s)^{2}\right\} & \geq \frac{1}{\frac{|\gamma|^{2}}{\sigma_{n}^{2}} s^{2} \operatorname{Re} \sum_{k_{x}^{\prime}, k_{y}^{\prime}}\left|2 O_{0}\left(s k_{x}^{\prime}, s k_{y}^{\prime}\right)+s k_{x}^{\prime} \frac{\partial O_{0}\left(s k_{x}^{\prime}, s k_{y}^{\prime}\right)}{\partial\left(s k_{x}^{\prime}\right)}+s k_{y}^{\prime} \frac{\partial O_{0}\left(s k_{x}^{\prime}, s k_{y}^{\prime}\right)}{\partial\left(s k_{y}^{\prime}\right)}\right|^{2}} \\
E\left\{(\hat{\beta}-\beta)^{2}\right\} & \leq \frac{1}{\frac{|\gamma|^{2}}{\sigma_{n}^{2}} s^{4} \operatorname{Re} \sum_{k_{x}^{\prime}, k_{y}^{\prime}}\left|s k_{y}^{\prime} \frac{\partial O_{0}\left(s k_{x}^{\prime}, s k_{y}^{\prime}\right)}{\partial\left(s k_{x}^{\prime}\right)}-s k_{x}^{\prime} \frac{\partial O_{0}\left(s k_{x}^{\prime}, s k_{y}^{\prime}\right)}{\partial\left(s k_{y}^{\prime}\right)}\right|^{2}}
\end{aligned}
$$




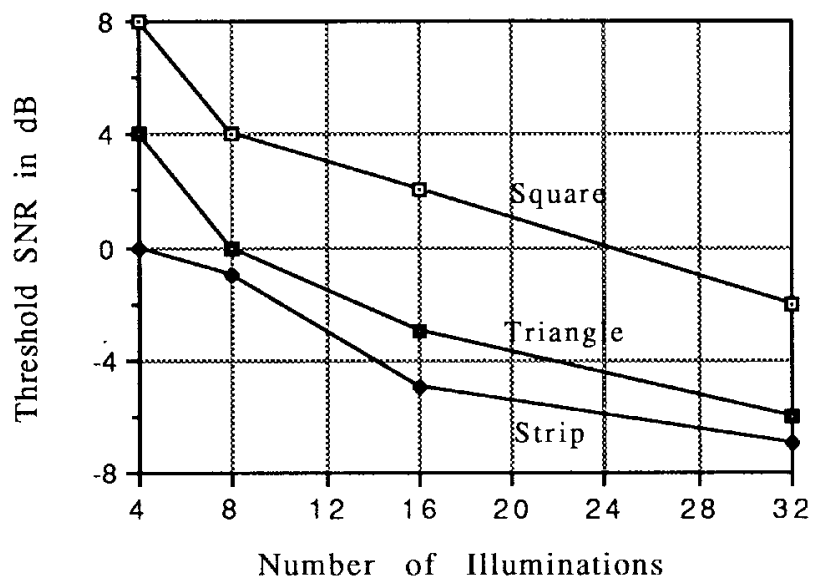

Fig. 3. Threshold SNR for at least $90 \%$ success in correct estimation of scale and orientation as a function of number of illuminations. Fifty Monte-Carlo runs were performed. The stripe requires lowest threshold SNR and the square hoghest. The triangle lies in between the cases.

TABLE II

Comparison of CRB's and MEAN SQuare ERror (MSE) For DifFerent ANGles of Orientation (Other PaRameters ARE $N_{L}=16, N_{R}=32, s=2$, AND SNR $=15.3 \mathrm{~dB}$ ).

The WaVelength of the Radiation is Assumed to be $1 \mathrm{~m}$

\begin{tabular}{c|l|l|l|l}
\hline $\begin{array}{c}\text { Orientation } \\
\text { angle } \\
(\beta)\end{array}$ & CRB for Scale/s ${ }^{2}$ & $\begin{array}{c}\text { MSE in } \\
\text { estimated } \\
\text { scale/s }\end{array}$ & $\begin{array}{c}\text { CRB for } \\
\text { orientation }\end{array}$ & $\begin{array}{c}\text { MSE in } \\
\text { estimated } \\
\text { orientation }\end{array}$ \\
\hline $0^{0}$ & $5.7089 \mathrm{e}-06$ & $5.9037 \mathrm{e}-06$ & $5.0642 \mathrm{e}-05$ & $5.1746 \mathrm{e}-05$ \\
\hline $10^{0}$ & $5.8043 \mathrm{e}-06$ & $7.0073 \mathrm{e}-06$ & $5.5717 \mathrm{e}-05$ & $6.4249 \mathrm{e}-05$ \\
\hline $20^{0}$ & $5.6221 \mathrm{c}-06$ & $6.0444 \mathrm{e}-06$ & $4.4946 \mathrm{e}-05$ & $4.9411 \mathrm{e}-05$ \\
\hline $40^{0}$ & $5.5518 \mathrm{e}-06$ & $6.5848 \mathrm{e}-06$ & $4.5063 \mathrm{e}-05$ & $6.1276 \mathrm{e}-05$ \\
\hline
\end{tabular}

TABLE III

Comparison of CRB's and Mean Square Error (MSE) For DifFerent Scales (Other Parameters Are $N_{L}=16, N_{R}=32, \beta=20^{\circ}$, AND SNR $=15.3 \mathrm{~dB}$ ). The WaVelength of the Radiation IS AsSUMED to Be $1 \mathrm{~m}$

\begin{tabular}{c|c|c|c|c}
\hline $\begin{array}{c}\text { Scale } \\
(\mathrm{s})\end{array}$ & $\begin{array}{c}\text { CRB for } \\
\text { Scale/ } / \mathrm{s}^{2}\end{array}$ & $\begin{array}{c}\text { MSE in } \\
\text { estimated } \\
\text { scale } / \mathrm{s}^{2}\end{array}$ & $\begin{array}{c}\text { CRB for } \\
\text { onientation }\end{array}$ & $\begin{array}{c}\text { MSE in } \\
\text { estimated } \\
\text { orientation }\end{array}$ \\
\hline 0.25 & $8.7134 \mathrm{e}-06$ & $9.2214 \mathrm{e}-06$ & $8.2711 \mathrm{e}-04$ & $9.5376 \mathrm{e}-04$ \\
\hline 0.5 & $8.3348 \mathrm{e}-06$ & $8.8571 \mathrm{e}-06$ & $1.3278 \mathrm{e}-04$ & $1.4008 \mathrm{e}-04$ \\
\hline 1.0 & $7.4335 \mathrm{e}-06$ & $7.9803 \mathrm{e}-06$ & $7.3486 \mathrm{e}-05$ & $1.0521 \mathrm{e}-04$ \\
\hline 2.0 & $5.6221 \mathrm{e}-06$ & $6.0445 \mathrm{e}-06$ & $4.4946 \mathrm{e}-05$ & $4.9411 \mathrm{e}-05$ \\
\hline 3.0 & $4.7221 \mathrm{e}-06$ & $5.0600 \mathrm{e}-06$ & $3.4231 \mathrm{e}-05$ & $3.6429 \mathrm{e}-05$ \\
\hline
\end{tabular}

\section{Discussion}

The CRB's for scale and orientation increase with wavelength. To explain this phenomenon, recall that as the wavelength increases, the size of the disc in the Fourier plane decreases, and hence, only a low-frequency part of the Fourier transform is available for shape estimation. The effect of orientation of the object has maximum effect in the high-frequency region, particularly for an equidimensional object such as a square. Therefore, for reliable estimation, the Fourier transform of the object over as large a disk as possible must be used. Conversely, the objects such as a strip or triangle having a significant angular variation of the spectrum are more likely to be correctly estimated. Thus, for a perfectly equidimensional object, such as a circle, it is not possible to estimate the orientation at all. For small objects (a fraction wavelength), the estimation error is higher because most of the high-frequency spectrum falls outside the disc.

\section{REFERENCES}

[1] K. Iwata and R. Nagata, "Calculation of three dimensional refractive index distribution from interferrograms," J. Opt. Soc. Amer., vol. 60, pp. 133-135, 1970.

[2] A. J. Devaney and G. A. Tsihirintzis, "Maximum likelihood estimation ofobject location in diffraction tomography," IEEE Trans. Signal Processing, vol. 39, pp. 672-682, 1991.

[3] G. A. Tsihirintzis and A. J. Devaney, "Maximum likelihood estimation of object location in diffraction tomography, Part II; strongly scattering objects," IEEE Trans. Signal Processing, vol. 39, pp. 1466-1470, 1991.

[4] _ , "Application of a maximum likelihood estimator in an experimental stuy in ultrasonic diffraction tomography," IEEE Trans. Med. Imag., vol. 12, pp. 545-554, 1993.

[5] D. J. Rossi and A. S. Willsky, "Reconstruction from projections based on detection and estimation of objects-Parts I \& II," IEEE Trans. Acoust., Speech, Signal Processing, vol. ASSP-32, pp. 886-906, 1984.

[6] _ "ML estimation of object size and orientation from projection data," in Proc. IEEE ICASSP, 1984, vol. 3.

[7] P. S. Naidu, A. Vasuki, P. Sathya Murthy, and L. Anand, "Diffraction tomographic imaging with a circular array," IEEE Trans. Ultrason. Ferroelect. Freq. Contr., vol. 42, pp. 787-789, 1995.

[8] A. J. Devaney and G. Beylkin, "Diffraction tomography using arbitrary transmitter and receiver surfaces," Ultrason. Imag., vol. 6, pp. 181-193, 1984.

[9] M. Abramowitz and L. Stegun, Eds., Handbook of Mathematical Functions. New York: NBS, 1964.

[10] W. H. Press, "Numerical recepies in Fortran," The Art of Scientific Computing. Cambridge, U.K.: Cambridge Univ. Press, 1992.

[11] H. L. Van Trees, Detection, Estimation and Modulation Theory, Part I. New York: Wiley, 1968.

[12] A Schatzberg, A. J. Devaney, and A. J. Wittens, "Estimating target location from scattered field data," Signal Process., vol. 40, pp. 227-237, 1994.

[13] D. J. Rossi, A. S. Willsky, and D. M. Spielman, "Object shape estimation from tomographic measurements-A performance analysis," Signal Process., vol. 18, no. 1, pp. 63-87, 1989.

\section{A Two-Level Interleaving Architecture for Serial Convolvers}

Francescomaria Marino

\begin{abstract}
In this correspondence, we present a bit-serial architecture for convolving/correlating long numerical sequences by long filter functions. Because of its two-level interleaving structure, the proposed device does not require "wait cycles" between consecutive input samples. As a result, it achieves the highest possible throughput. Cascadability, fault tolerance, feasibility in VLSI technology, and computing performances are discussed and analyzed.
\end{abstract}

Index Terms - Bit serial ASICS, convolvers/correctors, pipelined architectures.

\section{INTRODUCTION}

In this correspondence, we present a bit-serial VLSI architecture for the direct convolution/correlation of long numerical sequences by

Manuscript received March 9, 1998; revised October 1, 1998. The associate editor coordinating the review of this paper and approving it for publication was Dr. Konstantinos Konstantinides.

The author is with the Dipartimento di Elettrotecnica ed Elettronica, Facoltà di Ingegneria, Politecnico di Bari, Bari, Italy (e-mail: marino@poliba.it).

Publisher Item Identifier S 1053-587X(99)03249-3. 